This item is the archived peer-reviewed author-version of:

\title{
Bending gold nanorods with light
}

\section{Reference:}

Babynina Anastasia, Fedoruk Michael, Kühler Paul, Meledin Alexander, Döblinger Markus, Lohmüller Theobald.- Bending gold nanorods with light

Nano letters / American Chemical Society - ISSN 1530-6984 - (2016), p. 1-19

Full text (Publisher's DOI): http://dx.doi.org/doi:10.1021/ACS.NANOLETT.6B03029

To cite this reference: http://hdl.handle.net/10067/1351720151162165141 
This document is confidential and is proprietary to the American Chemical Society and its authors. Do not copy or disclose without written permission. If you have received this item in error, notify the sender and delete all copies.

\section{Bending Gold Nanorods with Light}

\begin{tabular}{|r|l|}
\hline Journal: & Nano Letters \\
\hline Manuscript ID & nl-2016-03029w.R1 \\
\hline Manuscript Type: & Communication \\
\hline Date Submitted by the Author: & 02-Sep-2016 \\
\hline Complete List of Authors: & $\begin{array}{l}\text { Babynina, Anastasia; LMU Munich, Physics } \\
\text { Fedoruk, Michael; Ludwig-Maximilians-Universtität München, Department } \\
\text { für Physik und CeNS } \\
\text { Kühler, Paul; LMU Munich, Physics } \\
\text { Meledin, Alexander; Antwerp University, Electron microscopy for materials } \\
\text { science (EMAT), Physics Department } \\
\text { Döblinger, Markus; LMU, Chemistry and Biochemistry } \\
\text { Lohmueller, Theobald; LMU Munich, Physics }\end{array}$ \\
\hline
\end{tabular}

SCHOLARONE

Manuscripts 


\title{
Bending Gold Nanorods with Light
}

\author{
Anastasia Babynina ${ }^{1}$, Michael Fedoruk ${ }^{1}$, Paul Kühler ${ }^{1}$, Alexander Meledin ${ }^{3}$, Markus Döblinger ${ }^{4}$, \\ Theobald Lohmüller ${ }^{1,2} *$ \\ ${ }^{1}$ Photonics and Optoelectronics Group, Department of Physics and Center for NanoScience \\ (CeNS), LMU München, Amalienstraße 54, Munich, 80799, Germany \\ ${ }^{2}$ Nanosystems Initiative Munich (NIM), Schellingstraße 4, 80539 Munich, Germany \\ ${ }^{3}$ EMAT, University of Antwerp, Groenenborgerlaan 171, 2020, Antwerp, Belgium \\ ${ }^{4}$ Department of Chemistry, LMU, München, Butenandtstr. 5-13 (E), 81377 Munich, Germany \\ KEYWORDS \\ Gold nanorods, plasmonic heating, optical forces, V-shaped nanoantennas
}

\begin{abstract}
V-shaped gold nanoantennas are the functional components of plasmonic metasurfaces, which are capable of manipulating light in unprecedented ways. Designing a metasurface requires the custom arrangement of individual antennas with controlled shape and orientation. Here, we show how highly crystalline gold nanorods in solution can be bend, one-by one, into a V-shaped geometry and printed to the surface of a solid support through a combination of plasmonic heating and optical force. Significantly, we demonstrate that both the bending angle and the orientation of each rod-antenna can be adjusted independent from each other by tuning the laser
\end{abstract}


intensity and polarization. This approach is applicable for the patterning of V-shaped plasmonic antennas on almost any substrate, which holds great potential for the fabrication of ultrathin optical components and devices.

Plasmonic metasurfaces can be engineered to control the amplitude, phase, and polarization of incident light by introducing an abrupt change of the optical properties at a sub-wavelength thin interface ${ }^{1-3}$. A well-studied example are metasurfaces made of 'V-shaped' gold nanoantennas ${ }^{4,5}$. These structures display two orthogonal plasmonic resonances, which are determined by the opening angle and the length of each antenna branch ${ }^{6,7}$. Both resonances can be excited by light with a corresponding wavelength and polarization and the combination of both modes allows to induce phase shifts between incoming and scattered light over the full range of $2 \pi^{4,8,9}$. The rational design metasurfaces, however, requires the precise patterning of individual V-shaped antennas with different geometries in a two-dimensional array ${ }^{3,10}$. Due to the small size and complexity of the antenna structures, this technologically challenging and demands the use of high-end nanofabrication methods, such as electron-beam lithography ${ }^{11}$.

Optical printing of plasmonic particles is an alternative to standard lithography approaches ${ }^{12,13}$. In recent years, the use of optical forces has emerged as a straightforward way to pattern spherical gold or silver nanoparticles with high lateral resolution ${ }^{14-16}$. For nanorods, it has been shown that optical printing can be used to position individual particles on any desired location on the surface of a glass cover slip and also that they can be aligned with respect to the laser polarization $^{17-19}$. The process is most efficient when the laser wavelength is resonant with the particle plasmon and generally applicable to a variety of particle shapes ${ }^{19-21}$. Conveniently, a 
plethora of particle morphologies and compositions are accessible by colloidal chemistry today ${ }^{22}$. The chemical synthesis of V-shaped particle, however, remains a challenge.

Alternatively, one could imagine to bend a single nanorod by the application of force and heat ${ }^{12}$, 23-25. Several groups have studied melting of gold nanorods with laser light in the past. In their seminal work, Link et al. have studied the shape transformation of gold nanorods in solution ${ }^{26-28}$. They found that the transition from a gold rod to a sphere starts at the interior of the rod by the creation of point and line defects, which eventually leads to the formation of planar stacking defects and twinning. This is followed by the surface diffusion of gold atoms from the tips to the center of the rod. Melting with a femtosecond laser led to the formation of spherical nanoparticles, while an abundance of other shapes was found for longer pulses that were interpreted to display an early stage of melting ${ }^{29}$. Also 'banana'- or 'sickle'-shaped geometries were observed by Link and a other groups ${ }^{30-32}$, yet the process how controlled nanorod deformation could be employed for the synthesis of $\mathrm{V}$-shaped plasmonic nanoparticles has not been investigated to date.

Here, we report how a combination of optical forces and plasmonic heating renders it possible to bend gold nanorods and simultaneously print them on the surface of a flat substrate. We demonstrate that the degree of bending and the alignment on the substrate of each individual Vshaped antenna is controllable by the amount of heat, force, and the polarization of the laser beam.

A schematic illustration of the experiment is shown in Figure 1a. Gold nanorods (124 x 21nm) were immersed in water and illuminated by a near infrared (NIR) laser beam $(\lambda=1064$, continuous wave (cw)) that was focused $2.8 \mu \mathrm{m}$ above the surface of a glass substrate (a more 
detailed description of experimental parameters is given in the "Dark-field spectroscopy and particle manipulation" section of the Supporting Information). The wavelength of the laser (1064nm) was chosen to be resonant with the longitudinal plasmon of the rod (SI: Figure S1). A single rod that is diffusing into the laser focus is therefore subject to strong scattering forces and pushed along the direction of the propagating light ${ }^{19}$ (Figure 1a). First, the gold rods were printed one-by-one by increasing the laser power and the resulting pattern was analyzed by scanning electron microscopy (SEM). For a laser power density of $0.45 \mathrm{MW} / \mathrm{cm}^{2}$, individual rods were deposited on the substrate with a preferred orientation along the polarization axis of the laser beam (Figure 1b, left inset*). After gradually increasing the power, however, the gold nanoparticles transformed from a straight to a bent shape (Figure $1 \mathrm{~b}$, right inset**). The bent structures were observed for a laser power density greater than $0.45 \mathrm{MW} / \mathrm{cm}^{2}$, which is sufficient to heat the particles above the melting point of gold ${ }^{33}$ (Figure 1b, red dotted line, SI: Figure S2). Although previous reports have shown that photo-thermal reshaping of rods to spheres can already happen below the melting temperature ${ }^{34,35}$, we rarely observed any particle reshaping in this case, likely due to the short duration of the printing step $(\sim \mathrm{ms})$. Analyzing the degree of bending as a function of the laser intensity also showed that the bending angle of the rods is adjustable from $180^{\circ}$ to almost $90^{\circ}$ (Figure $1 \mathrm{~b}$ ). Changing the power during the process for each rod thus allows for sequential printing of particles with different opening angles onto the substrate. As an example, a series of SEM images of bent gold nanorods with different bending angles are shown in Figure 1c.

The optical properties of bent rods were characterized by dark-field scattering spectroscopy. Examples of the polarization dependent scattering spectra for a V-shaped particle with an opening angle of $104^{\circ}$ are shown in Figure 2a. The bent nanorod displays two plasmon 
resonances at $\sim 700 \mathrm{~nm}$ and $\sim 1000 \mathrm{~nm}$ that correspond to the transversal or 'symmetric' and the longitudinal or 'antisymmetric' resonances with respect to the symmetry axis of the structure ${ }^{4,6 \text {, }}$ ${ }^{9}$. Only the symmetric resonance is observed when the laser polarization was oriented parallel to the symmetry axis of the structure. On the other hand, the antisymmetric resonance is excited if the polarization is turned by $90^{\circ}$. The polarization dependence of the scattering spectrum of a single particle is shown in Figure 2b. We found that the measured data was in good agreement with FDTD simulations (Figure 2c, d). Notably, the peak position is depending on the opening angle of the nanostructure. Compared to a straight rod, the transverse mode at $560 \mathrm{~nm}$ is shifted to the red spectra region. In comparison, the longitudinal plasmonic peak shifts to the blue region of the spectrum due to a reduction of the effective dipole length of the plasmon propagation (SI: Figure S3).

The change of the nanorod geometry is an indication for laser induced melting. We performed high-resolution 'High Angle Annular Dark Field Scanning Transmission Electron Microscopy' (HAADF STEM) imaging of both straight and bent rods to investigate the crystal structure after the laser irradiation. As shown in Figure 3a, b, the non-deformed rods and the branches of the bent rods are elongated parallel to a $<100>$ direction. Straight rods display a high order of crystallinity with almost no visible defects and no signs of melting or reshaping (Figure 3a, SI: Figure S5). The STEM images of bent structures show twin domains that are formed in the center of the rod (Figure 3b-d). The domains display the thermodynamically stable twin grain boundaries along the $\{111\}$ planes. This observation is in good agreement with the findings of previous studies $^{27}$ and with molecular dynamic simulations of FCC nanowires, reporting that the deformation in a single FCC crystal is possible due to twin formation along the $\{111\}$ crystal planes $^{36}$. This reorganization of gold atoms in the nanorod center could account for the kinked or 
curved shape of the final structure. However, it does not fully explain the laser power dependence of the bending angle. Furthermore, bending was only observed for rods in solution and not for those attached to the substrate. These particles were molten into spherical or other undefined shapes instead (SI, Figure S6). This suggests that also further experimental parameters, such as the effect of optical forces acting on the gold rods have to be considered.

A gold nanorod aligns perpendicular to the Poynting vector when it diffuses into the laser beam $^{18,37}$. In this position, the rod is pushed in the direction of the beam propagation by the optical force ${ }^{17,} 19$. This causes hydrodynamic pressure on the rod, which is working in the opposite direction of its movement ${ }^{38}$. We calculated the distribution of the hydrodynamic pressure along the long axis of a horizontally aligned nanorod by numerically solving the NavierStokes equations (Figure 4a). The results indicate that the pressure is almost $30 \%$ stronger at the tips compared to the center. Assuming that the heated rods are to some degree soft and deformable, this pressure difference then leads to a symmetric deformation of the linear structure. The bending occurs primarily in the center region of the rod, which is in good agreement with our experimental findings. As already mentioned, no bending was observed for rods that were attached to the substrate, potentially since no movement of the particles was involved during heating (SI: Figure S6). Hydrodynamic pressure can also explain the bending angle dependence on the laser intensity. With high laser powers, both the particle temperature and optical forces are increasing. The nanorods thus remain deformable over a distance of several micrometers away from the laser focus and are, at the same time, subject to a stronger hydrodynamic pressure. Both factors therefore result in a stronger bending deformation.

Finally, the particles undergo a considerable change of their plasmonic properties. Both, the peak positions of the absorption and scattering cross-section of the bent structures are decreasing and 
blue-shifted for a smaller bending angle. That leads to a less efficient heating and weaker optical forces. For strong bending deformation, the particle temperature could eventually drop below the melting point, which causes the particle to freeze instantaneously. An example for this behavior is shown in Figure 4c. A straight nanorod is heated above melting temperature within a range of $\pm 200 \mathrm{~nm}$ from the laser focus for a laser power density of $0.45 \mathrm{MW} / \mathrm{cm}^{2}$. The optical force at this point is about $3.5 \mathrm{pN}$. For $0.75 \mathrm{MW} / \mathrm{cm}^{2}$ the range is already increased to approximately \pm $800 \mathrm{~nm}$ and furthermore to $\sim \pm 1.5 \mu \mathrm{m}$ for a laser power density of $2.11 \mathrm{MW} / \mathrm{cm}^{2}$. The scattering forces, however, are still in the $\mathrm{pN}$ range and thus high enough to print bent particles to the underlying substrate. As mentioned earlier, a maximum bending angle around $90^{\circ}$ was found in our experiments. We believe that this limitation is largely a consequence of the interplay between the position of the laser focus in our setup and the overall laser power.

In our setup, the laser was focused only $2.8 \mu \mathrm{m}$ above the glass substrate. A too high laser power can thus result in continuous heating of the particle even after it is printed to the glass surface. In this case, the branches of the V-shaped particle start to melt at the tips, which also shortens both antenna arms. This also leads to a further blue shift of the longitudinal plasmon mode, which is independent of the bending angle (an example is shown in Figure S4 of the Supporting Information).

Overall, the degree of nanorod bending that is observed for different laser intensities is a result of an interplay of both, the hydrodynamic pressure acting on the rod and the change of the optical properties with increasing nanorod deformation. We therefore believe that a critical aspect to achieve this controlled deformation was using the $\mathrm{cw}$ light source instead of a pulsed laser. The nanorods are continuously heated and pushed while they are interacting with the laser beam. The 
continuous melting and particle movement allows to have nanorod re-shaping and hydrodynamic interactions at the same time.

In the last step, we looked into the possibility to align and bend the structures at the same time by laser printing. An example for a line of $\mathrm{V}$-shaped particles and the corresponding polarization direction of the laser beam is shown in Figure 5a. We performed a statistical analysis of the orientation of printed nanorods and bent particles and compared the values. The angular deviation of the particle alignment from the normal orientation for all three cases is shown in Figure $5 \mathrm{~b}-\mathrm{d}$. For normal gold rods, an orientation precision of $\pm 26^{\circ}$ was achieved (Figure $5 \mathrm{~b}$ ). For the bent rods, the orientation precision was $\pm 20^{\circ}$ (Figure $5 \mathrm{c}$ ) and $\pm 30^{\circ}$, depending on the bending angle (Figure 5d). These results illustrate that a preferential alignment of the particles is feasible, although the precision appears to become slightly more inaccurate as the bending deformation of the structure is increased. These values, however, could likely be improved by using a two-color laser printing approach by Do et al. ${ }^{19}$, where an orientation precision of $\pm 16^{\circ}$ was achieved for optical printing of gold rods. As shown in Figure 5a, the orientation of the opening angle was either to the left or to the right with respect to the polarization axis, because the rods can fall on both sides with the same probability as soon as they touch the substrate. Modifying the shape of the laser beam or tilting the substrate on one side are possible strategies that that will likely allow to have a better control over the angle orientation, which will be subject to future investigations.

In conclusion, we have demonstrated that gold nanorods can be bent in a controlled way by means of light. The combination of optical forces and plasmonic heating renders it possible to adjust the bending angle of single gold nanorods by changing the laser power. Nanorod bending has immediate consequences on the plasmonic properties of the particles, which, along with 
hydrodynamic pressure, determines the degree of deformation. Importantly, bending was only observed for particles that were freely diffusing in solution. This illustrates that a combination of particle movement and melting transformation is required to control the overall process. Furthermore, we could show that straight as well as bent particles can be printed optically onto a glass substrate. Using polarized laser light allows controlling the alignment of the nanorods on the surface. This new approach renders it possible to generate arrays of bent or 'V-shaped' nanoantennas and to assemble them in a controlled orientation on top of a solid support, which holds great potential for the fabrication of flat optics and metasurfaces in the future.

Supporting Information Available: The following material is included as supporting information: A description of materials and methods, temperature simulations, supporting scattering data, FDTD calculations, supporting HRTEM and SEM micrographs, hydrodynamics simulations. This material is available free of charge via the Internet at http://pubs.acs.org.

\section{Corresponding Author}

* TL: t.lohmueller@Imu.de

Funding Sources

The authors acknowledge financial support by the DFG through the Nanosystems Initiative Munich (NIM) and through the Collaborative Research Center (SFB1032), project A8.

\section{ACKNOWLEDGMENT}

We would also like to thank Prof. Jochen Feldmann and Bernhard Bohn for fruitful discussions.

\section{Notes.}

The authors declare no competing financial interests. 
a

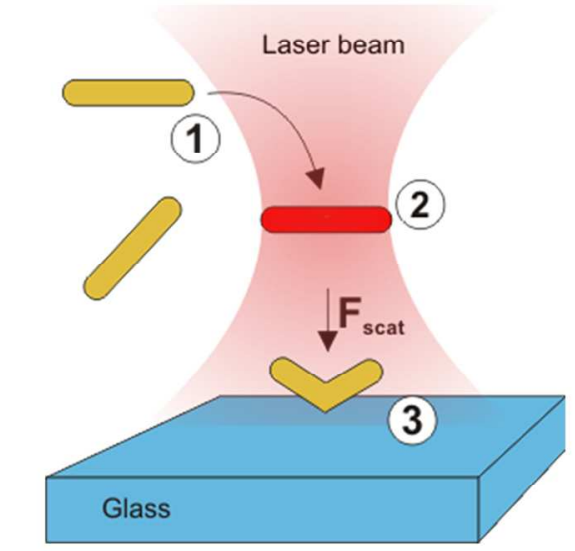

C

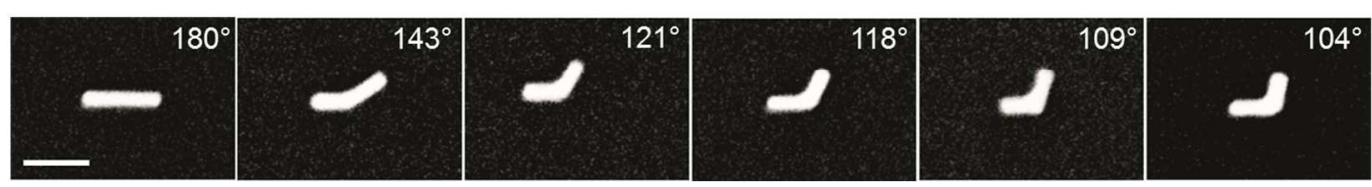

b

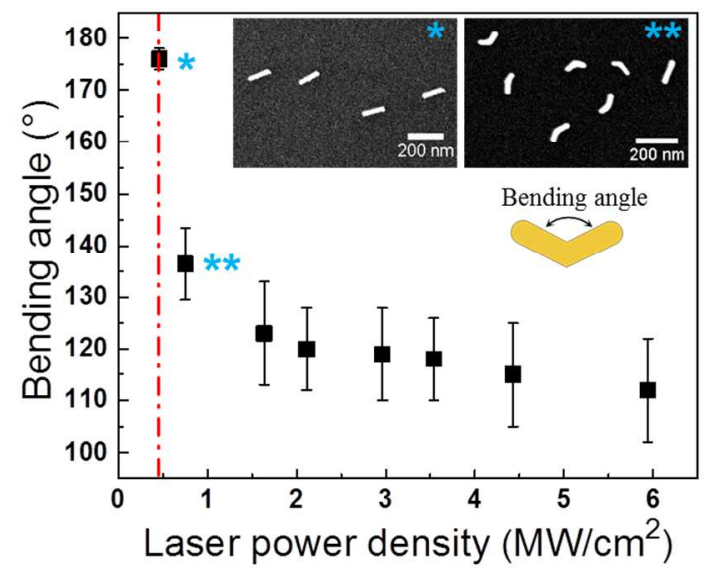

Figure 1. Optical bending and printing of gold nanorods. a, Schematic illustration of the bending process. Gold nanorods are diffusing in solution (step 1). A single rod aligns horizontally as soon as it enters the laser focus (step 2). The nanoparticle is heated in the laser beam, while optical forces simultaneously push the particle towards the substrate (step 3) b, The bending angle of the printed particles can be controlled by the laser power density. Optically printed gold nanorods display a transition from a straight $(*)$ to a bent $(* *)$ morphology above a laser power density of $0.45 \mathrm{MW} / \mathrm{cm} 2$. The red dotted line indicates the laser power density that is required to heat the nanorods above their melting temperature according to simulations. Error bar represents the standard deviation from mean value. For each laser power density, at least 15 bent gold nanorods were measured. c, SEM images of gold nanorods that were printed with different bending angles. Scale bar is $100 \mathrm{~nm}$. 
a

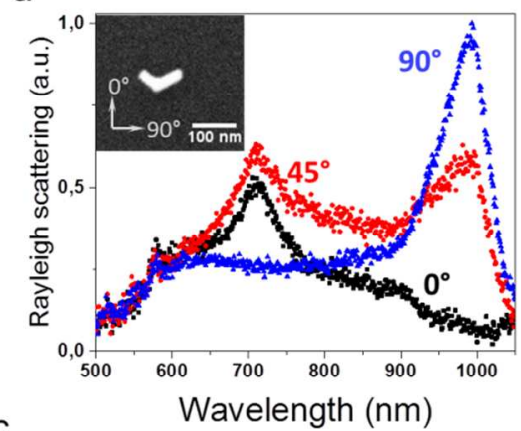

C
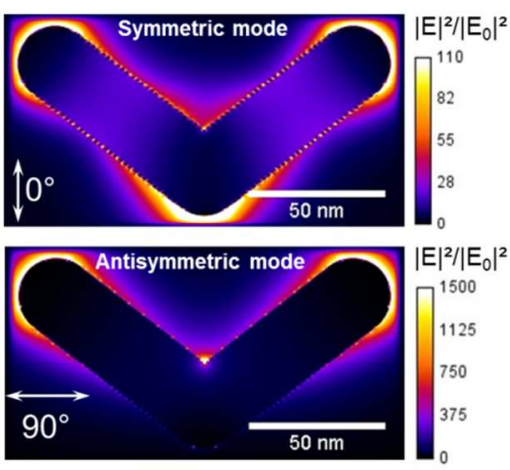

b

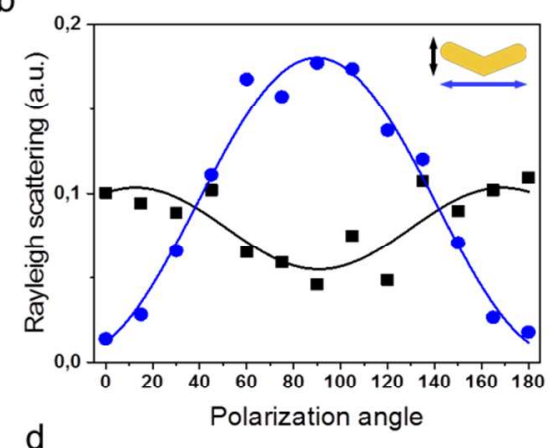

d

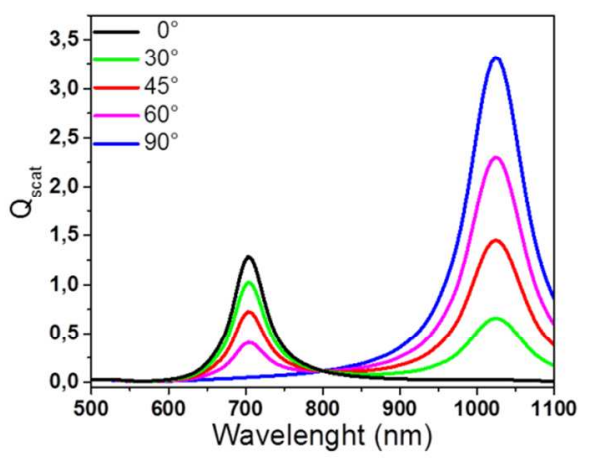

Figure 2. Optical properties of bent gold nanorods. a, Polarization-dependent Rayleigh scattering spectra of a single V-shaped gold particle in water. The bending angle of the structure is $104^{\circ}$. Two peaks with a maximum of $700 \mathrm{~nm}$ and $1000 \mathrm{~nm}$ are observed that correspond to a 'symmetric' (black curve) and an 'antisymmetric' (blue curve) plasmon resonance with respect to the symmetry axis of the structure. Both modes can be separately excited with polarized light. Both plasmon resonances are visible if the particle is excited with polarized light at a $45^{\circ}$ angle (red curve). A SEM image of the bent gold nanorod and the corresponding alignment of the particle with respect to the laser polarization is shown in the upper right corner. The rod shows a slight deviation compared to a perfect V-shape, which manifests in a small background scattering visible in the angle dependent spectra. b, Polarization dependence of both peaks. c, FDTD simulations of the near-field enhancement and charge distribution in a bent gold nanorod particle for symmetric and antisymmetric excitation with polarized light. The colored scale bars present the enhancement factor of electromagnetic field with respect to initial incident light. d, 
Simulations of the polarization dependent scattering spectra of a bent gold nanorod with $105^{\circ}$ opening angle. The simulations show good agreement with the measured data. 


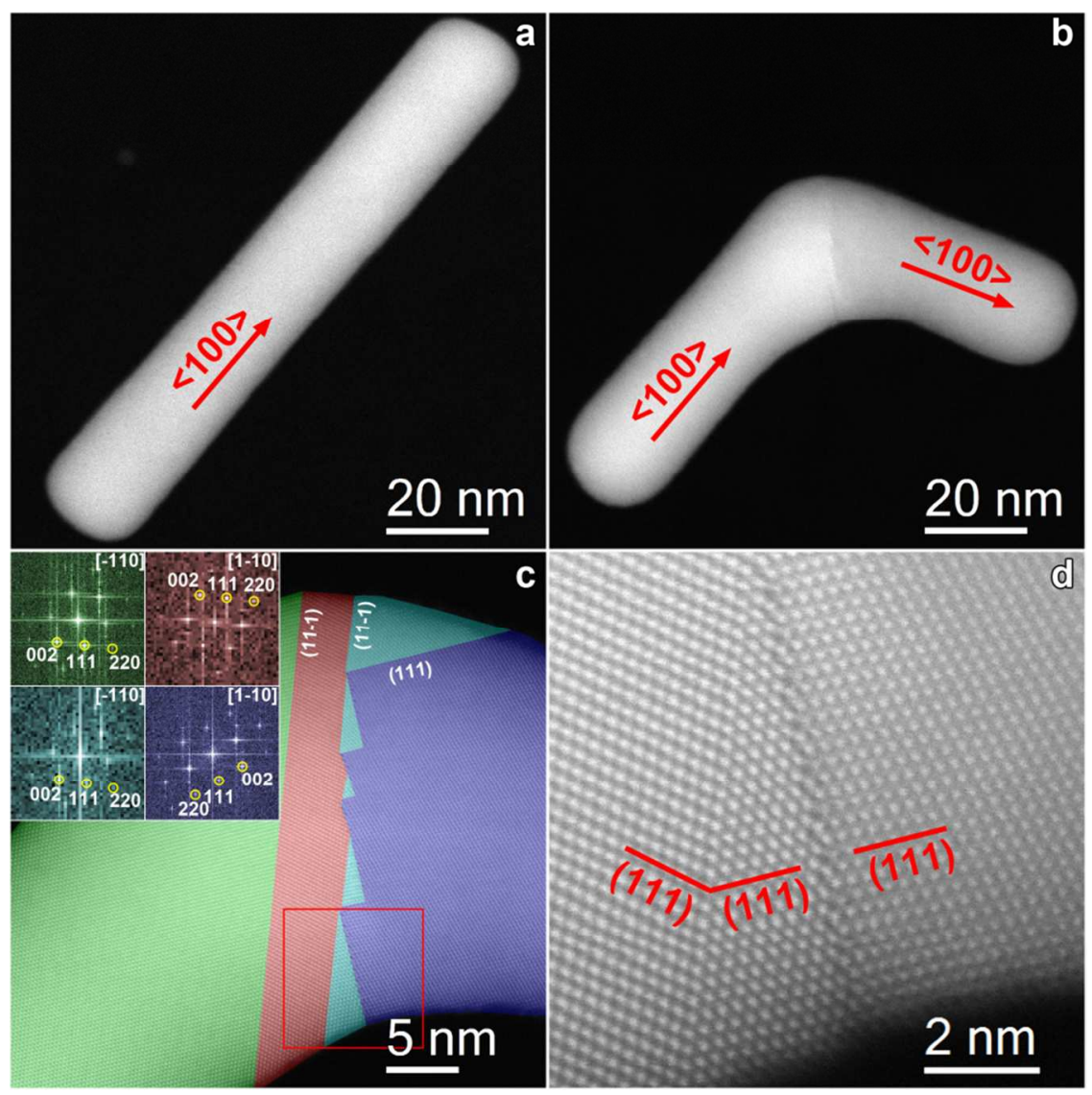

Figure 3. STEM study of crystal changes in V-shaped nanoantennas. a, STEM image of a straight gold nanorod. The rod is elongated parallel to the $<100>$ direction. No defects in the crystal structure are observed. b, STEM image of a bent gold nanorod. The branches of the nanorod still display the crystallographic orientation of a straight rod. c, Colored STEM image of twin domains (twin planes indicated in white) in the bent area with corresponding (color code) FFT. The green and cyan areas have equivalent crystal orientation. The red square shows the area magnified in (d). d, High resolution HAADF-STEM image of the twin structure (marked by the tilted $\{111\}$ planes). After printing, the bent nanorods remain crystalline. However, four types of monocrystalline differently oriented domains are observed at the kink of the rod. 
a

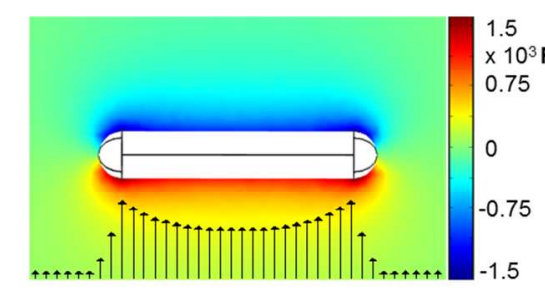

C Temperature $\left({ }^{\circ} \mathrm{C}\right)$

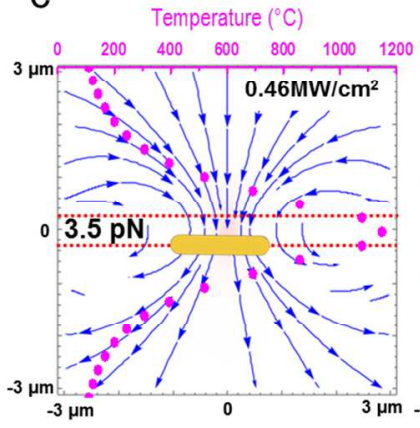

b

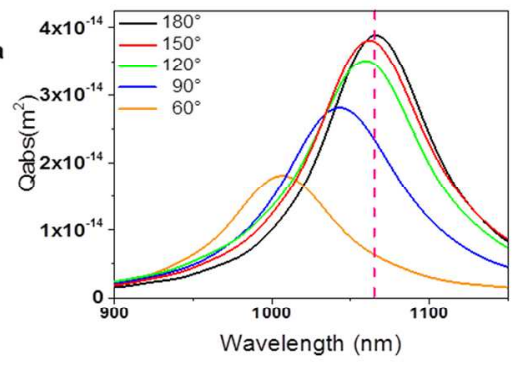

Figure 4. Hydrodynamic pressure and optical forces acting on a gold nanorod. a, Simulation of the hydrodynamic pressure along a gold nanorod that is moving in water at a speed of $0.02 \mathrm{~m} / \mathrm{s}$ (please see supporting information for further details). The line of black arrows highlights the relative intensities of the pressure at different positions along the rod. A higher hydrodynamic pressure is observed at the tips of compared to the middle part. $b$, FDTD simulations of the absorption cross sections of gold nanorods with different bending angles. A smaller bending angle leads to a decrease of the absorption cross section and a blue shift of the absorbance maximum, which results in less efficient heating at $\lambda=1064 \mathrm{~nm}$. c, Simulations of the scattering force for different laser power densities. The red dashed lines in all three plots represent the position where the calculated particle temperature is the melting temperature of gold. The corresponding scattering force at this position is shown for each plot. An example for the temperature of a straight gold nanorod at different positions along the laser beam is shown in the left panel. Absorption cross-sections of $3.88 \times 10^{-14}, 3.79 \times 10^{-14}$, and $3.47 \times 10^{-14} \mathrm{~m}^{2}$ were used to calculate the temperature for rods with a bending angle of $180^{\circ}, 150^{\circ}$, and $120^{\circ}$, respectively. 
Figure 5. Orientation control by optical printing with linear polarized light. a, SEM image of a line of five optically printed, V-shaped nanorods. b-d, Histogram analysis of the nanorod orientation of the printed nanorods with respect to the laser polarization. The ' 0,0 ' direction depicts the orientation of the polarized laser beam. 50 nanoparticles were measured for each histogram. b, Angular deviation of straight nanorods printed with polarized light. c, Angular deviation of bent nanorods with opening angle of $136^{\circ}$, and $\mathrm{d}$, angular deviation of bent nanorods with an opening angle of $118^{\circ}$. 


\section{REFERENCES}

1. Kildishev, A. V.; Boltasseva, A.; Shalaev, V. M. Science 2013, 339, 1232009.

2. Yu, N. F.; Capasso, F. Nature Materials 2014, 13, 139-150.

3. Meinzer, N.; Barnes, W. L.; Hooper, I. R. Nature Photonics 2014, 8, 889-898.

4. Yu, N. F.; Genevet, P.; Kats, M. A.; Aieta, F.; Tetienne, J. P.; Capasso, F.; Gaburro, Z. Science 2011, 334, 333-337.

5. Ni, X. J.; Emani, N. K.; Kildishev, A. V.; Boltasseva, A.; Shalaev, V. M. Science 2012, $335,427-427$.

6. Blanchard, R.; Aoust, G.; Genevet, P.; Yu, N. F.; Kats, M. A.; Gaburro, Z.; Capasso, F. Physical Review B 2012, 85, (15).

7. Stokes, N.; Cortie, M. B.; Davis, T. J.; McDonagh, A. M. Plasmonics 2012, 7, 235-243.

8. Kats, M. A.; Genevet, P.; Aoust, G.; Yu, N. F.; Blanchard, R.; Aieta, F.; Gaburro, Z.; Capasso, F. P. Natl Acad Sci USA 2012, 109, 12364-12368.

9. Bohn, B. J.; Schnell, M.; Kats, M. A.; Aieta, F.; Hillenbrand, R.; Capasso, F. Nano Letters 2015, 15, 3851-3858.

10. Yu, N. F.; Capasso, F. J Lightwave Technol. 2015, 33, 2344-2358.

11. Klein, M. F. G.; Hein, H.; Jakobs, P. J.; Linden, S.; Meinzer, N.; Wegener, M.; Saile, V.; Kohl, M. Microelectron Eng. 2009, 86, 1078-1080. 
12. Urban, A. S.; Carretero-Palacios, S.; Lutich, A. A.; Lohmuller, T.; Feldmann, J.; Jackel, F. Nanoscale 2014, 6, 4458-4474.

13. Urban, A. S.; Lutich, A. A.; Stefani, F. D.; Feldmann, J. Nano Letters 2010, 10, 47944798.

14. Guffey, M. J.; Scherer, N. F. Nano Letters 2010, 10, 4302-4308.

15. Gargiulo, J.; Cerrota, S.; Cortes, E.; Violi, I. L.; Stefani, F. D. Nano Letters 2016, 16, 1224-1229.

16. Bao, Y.; Yan, Z. J.; Scherer, N. F. J Phys Chem C 2014, 118, 19315-19321.

17. Ling, L.; Guo, H. L.; Zhong, X. L.; Huang, L.; Li, J. F.; Gan, L.; Li, Z. Y. Nanotechnology 2012, 23, 215302.

18. Selhuber-Unkel, C.; Zins, I.; Schubert, O.; Sonnichsen, C.; Oddershede, L. B. Nano Letters 2008, 8, 2998-3003.

19. Do, J.; Fedoruk, M.; Jackel, F.; Feldmann, J. Nano Letters 2013, 13, 4164-4168.

20. Huergo, M. A.; Maier, C. M.; Castez, M. F.; Vericat, C.; Nedev, S.; Salvarezza, R. C.; Urban, A. S.; Feldmann, J. Acs Nano 2016, 10, 3614-3621.

21. Guffey, M. J.; Miller, R. L.; Gray, S. K.; Scherer, N. F. Nano Letters 2011, 11, 40584066.

22. Sau, T.; Rogach., A. L. Colloidal Synthesis of Noble Metal Nanoparticles of Complex Morphologies, in Complex-Shaped Metal Nanoparticles: Bottom-Up Syntheses and Applications; Wiley-VCH: Weinheim, 2012. 
23. Baffou, G.; Quidant, R.; Girard, C. Appl. Phys. Lett. 2009, 94, 153109.

24. Dienerowitz, M.; Mazilu, M.; Dholakia, K. J Nanophotonics 2008, 2, 021875.

25. Govorov, A. O.; Richardson, H. H. Nano Today 2007, 2, 30-38.

26. Link, S.; Burda, C.; Mohamed, M. B.; Nikoobakht, B.; El-Sayed, M. A. J Phys Chem A 1999, 103, 1165-1170.

27. Link, S.; Wang, Z. L.; El-Sayed, M. A. J Phys Chem B 2000, 104, 7867-7870.

28. Link, S.; Burda, C.; Nikoobakht, B.; El-Sayed, M. A. Chem Phys Lett. 1999, 315, 12-18.

29. Link, S.; Burda, C.; Nikoobakht, B.; El-Sayed, M. A. J Phys Chem B 2000, 104, 61526163.

30. DeSantis, C. J.; Huang, D.; Zhang, H.; Hogan, N. J.; Zhao, H.; Zhang, Y.; Manjavacas, A.; Zhang, Y.; Chang, W.-S.; Nordlander, P.; Link, S.; Halas, N. J. J.Phys. Chem. C 2015, 151009142744004

31. Gordel, M.; Olesiak-Banska, J.; Matczyszyn, K.; Nogues, C.; Buckle, M.; Samoc, M. Phys. Chem. Chem. Phys. 2014, 16, 71-78.

32. Horiguchi, Y.; Honda, K.; Kato, Y.; Nakashima, N.; Niidome, Y. Langmuir 2008, 24, 12026-12031.

33. Buffat, P.; Borel, J. P. Phys Rev A 1976, 13, 2287-2298.

34. Petrova, H.; Juste, J. P.; Pastoriza-Santos, I.; Hartland, G. V.; Liz-Marzan, L. M.; Mulvaney, P. Phys. Chem. Chem. Phys. 2006, 8, 814-821. 
35. Taylor, A. B.; Siddiquee, A. M.; Chon, J. W. M. Acs Nano 2014, 8, 12071-12079.

36. Park, H. S.; Gall, K.; Zimmerman, J. A. J. Mech. Phys. Solids 2006, 54, 1862-1881.

37. Tong, L. M.; Miljkovic, V. D.; Kall, M. Nano Letters 2010, 10, 268-273.

38. Manghi, M.; Schlagberger, X.; Kim, Y. W.; Netz, R. R. Soft Matter 2006, 2, 653-668.

\section{Table of content graphic}
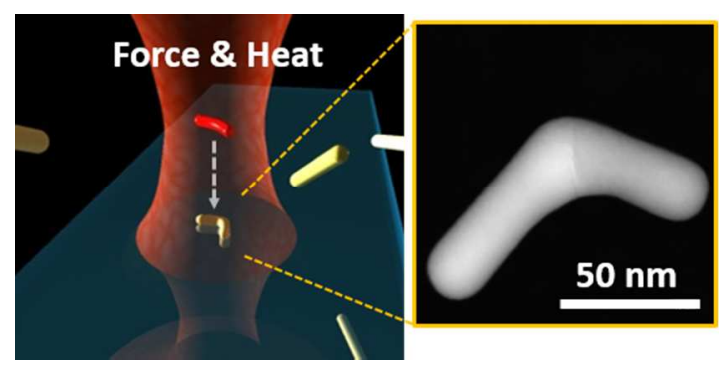